Introduction: Immediate breast reconstructions (IBR) have become an integral part of modern breast cancer management. However, in a small breast unit the spectrum of methods used for IBR could be limited, which could result in poorer results in some cases. The aim of the study is to evaluate the patient satisfaction and aesthetic outcome results in a breast unit where only implant-based IBR were performed.

Material and methods: During 20092016, 64 cases of implant-based IBR were performed in the university hospital. 55 patients completed the questionnaire and 38 underwent evaluation by a plastic surgeon. 33 skin-sparing and 22 nipple-sparing mastectomies were included. The study included 30 two-stage expander/implant and 25 direct-toimplant IBR cases.

Results: Overall satisfaction was reported by $89 \%$ of respondents. $93 \%$ were satisfied with appearance in clothes and $82 \%$ with appearance in a bra. There was a significant difference with satisfaction in nude appearance between groups with a removed (3\%) and a spared nipple (46\%). The plastic surgeon evaluated overall outcome as satisfactory in $61 \%$ and poor in $39 \%$. Spearman coefficient showed a moderate negative correlation between body mass index (BMI) and aesthetic outcome $(p=0.02)$, as well as BMI and volume differences between breasts $(p=0.03)$. Patients evaluated their breast symmetry as satisfactory in $55 \%$, and the plastic surgeon concluded the same in 55\% of 38 cases.

Conclusions: Most of the patients were satisfied with the aesthetic outcome of IBR. Nipple preservation considerably improved satisfaction rates. However, implant-based IBR revealed suboptimal cosmetic results in the subset of cases with increased $\mathrm{BMI}$ and other IBR methods should be considered in those cases.

Key words: immediate breast reconstruction, immediate breast reconstruction, breast cancer.

Contemp Oncol (Pozn) 2019; 23 (2): 96-99 DOI:https://doi.org/10.5114/wo.2019.8588

\section{Body mass index and nipple preservation are major contributors to satisfaction and aesthetic outcome rates after implant-based immediate breast reconstruction}

\author{
Stanislavs Ukleikins ${ }^{1}$, Arvids Irmejs ${ }^{2,3}$, Ansis Gilis ${ }^{2}$, Elina Tauvena ${ }^{1}$, \\ Jelena Maksimenko ${ }^{2,3}$, Genadijs Trofimovics ${ }^{2}$, Janis Gardovskis ${ }^{1}$ \\ 'Department of Surgery, Rīga Stradiņš University, Riga, Latvia \\ ${ }^{2}$ Breast Unit, Paul's Stradiṇš Clinical University Hospital, Riga, Latvia \\ ${ }^{3}$ Institute of Oncology, Riga Stradiņš University, Riga, Latvia
}

\section{Introduction}

Immediate breast reconstructions (IBR) has become an integral part of modern breast cancer management. However, in a small breast unit spectrum of methods used for IBR could be limited, which in turn could result in suboptimal results in some cases.

The results of previously conducted studies showed that after delayed reconstruction women more often experience anxiety and distress, both before surgery and during the time between mastectomy and reconstruction. Due to the positive psychological influence and equal oncological safety of immediate breast reconstruction (IBR) in comparison to delayed reconstruction, the use of this method should be strongly considered [1, 2]. The aim of the study is to evaluate the patient satisfaction and aesthetic outcome results in a small breast unit where only implantbased IBR were performed.

\section{Material and methods}

During 2009-2016 in 64 breast cancer cases implant-based IBR were performed in a breast unit of a university hospital with 150-200 breast cancers treated annually. Before 2009 only several exceptional cases of IBR were performed in Latvia. In 3/64 (5\%) cases implants were removed because of infection and those patients were excluded from the study. At the time of evaluation (2016-2017) 2 patients died, and 4 patients refused to participate.

55 patients with IBR were included in the study and completed the questionnaire. The mean age of the study group was $47.7 \pm$ standard deviation (SD) of 9.8 years. The youngest patient was 27 years old and the oldest patient was 74 years old. Mean reported body mass index (BMI) at the time of evaluation was $24.9 \pm$ SD of 3.9. 33 skin sparing and 22 nipple sparing mastectomies were performed, 42 unilateral and 13 bilateral. Median time from reconstruction to evaluation was 47 months, but the shortest follow-up period was 8 months. In 25 cases one stage direct to implant IBR was carried out, but in another 30 cases two stage IBR was undertaken - during the cancer surgery IBR was done with tissue expander and at a later stage the tissue expander was replaced with a permanent implant. Ptosis of opposite breast was present in $42 \%$ of cases.

Only anatomic shape silicone gel implants by Eurosilicone or Allergan were used. In all cases the implant was in the full submuscular position. Sentinel node biopsy (SNB) was performed in 45 cases and axillary lymph node dissection (ALND) was performed in 10 cases. Neoadjuvant chemotherapy (NAC) was used in 34 cases and adjuvant radiation therapy in 17 cases. 
On the basis of similar studies [3-5] a new questionnaire was developed, which included 2 parts: overall satisfaction, aesthetic satisfaction and influence of surgery on the private life. The questionnaire consisted of 15 questions, 4 of which were YES/NO questions and in another 11 questions respondents could choose numeric values from 5 to 1, where 5 was considered as "very good" and 1 "very bad". In the result section answers with numeric values 5 to 3 were together classified as a good outcome, but answers with numeric values 1 and 2 were classified together as a poor outcome.

38 patients agreed to undergo evaluation by an independent plastic surgeon, which measured the three following criteria: aesthetic assessment (good, satisfactory, unsatisfactory), symmetry (good, satisfactory, unsatisfactory) and volume difference (none or minimal [ $<15 \%]$, mild [15-30\%], severe [>30\%]). Data on some additional visual parameters such as deforming scar and opposite breast ptosis were also collected for analysis.

All patients signed informed consent for participation in the study. Hospital permission was obtained to conduct the research and the study was approved at Riga Stradins University Ethics committee.

For information analysis MS Excel 2013 and IBM SPSS 24.0. were used. To analyze information descriptive statistics were used, including the $\chi^{2}$ test for categorical independent analysis, the Mann-Whitney test for comparing means and Spearman correlation coefficient and regression analysis for correlation determination between nonparametric data. All statistical differences were considered statistically significant if $p<0.05$.

\section{Results}

Overall $89 \%$ of respondents were satisfied with the reconstruction outcome. As many as $91 \%$ would recommend breast reconstruction surgery to a friend. Detailed general satisfaction and attitude towards the IBR procedure are summarized in Table 1.

In the case of intensive exercise further analysis revealed that complaints were associated significantly with the extent of axillary surgery. If SNB was performed, $45 \%$ of patients were complaint free, but after ALND - only $10 \%$.

During detailed aesthetic evaluation, 93\% were satisfied with appearance in clothes and $82 \%$ with appearance in a bra. There was a statistically significant difference with satisfaction in nude appearance between groups with a removed (3\%) and a spared nipple (46\%) respectively $(p<0.001)$. A summary of detailed aesthetics of satisfaction can be seen in Table 2. The internal consistency of the questionnaire was assessed by Cronbach's $\alpha$ test ( $\alpha=0.91)$.

An independent plastic surgeon evaluated overall outcome as satisfactory in $61 \%$ and poor in $39 \%$. In further analysis Spearman correlation coefficient showed a moderate negative correlation between body mass index (BMI) and aesthetic outcome $(p=0.02)$, as well as BMI and volume differences between breasts ( $p=0.03$ ) (Figs. 1 and 2). The plastic surgeon rated breast symmetry as satisfactory in $55 \%$ of 38 cases, and also patient self-assessment symmetry was reported as positive in 55\% of 55 cases.

In the plastic surgeon's opinion $71 \%$ of patients had no volume difference $(<15 \%)$ between breasts, $16 \%$ had a moderate (15-30\%) and $13 \%$ had a great (> 30\%) volume difference. Deforming scar was diagnosed by a plastic surgeon in $32 \%$ of cases; all of them underwent 2-stage expander-to-implant reconstruction. Level of satisfaction in nude appearance and good symmetry evaluation by the plastic surgeon strongly increased in later cases compared to earlier ones (Figs. 3 and 4). Those data confirm the existence of a learning curve in the case of IBR.

\section{Discussion}

In this study overall satisfaction was high, which can partially be explained by the relatively recent appearance of IBR in Latvia, and availability of the procedure itself plays an important role in regard to patient satisfaction in comparison to mastectomy alternative. Obtained data were similar to the Qureshi et al. results, where average satisfaction after direct-to-implant reconstruction was $80 \%$, but after two-step reconstructions 66\% [6].

According to our study results almost all women who underwent IBR did not regret that decision and would recommend it to a friend if needed regardless of used reconstruction technique. In published studies those results vary from $85 \%$ to $90 \%$ in implant-based reconstructions and up to $92 \%$ in reconstructions with tissue flaps $[4,7,8]$.

Discomfort during strenuous physical activities was associated with axillary treatment extent. According to our data more than a half of patients who underwent implant-based reconstruction had discomfort during strenuous physical activities. Considerably fewer patients had minor discomfort during everyday activity. These results correspond to research results of Dieterich et al., where on

Table 1. General satisfaction and attitude toward immediate breast reconstructions $(N=55)$

\begin{tabular}{|c|c|c|c|c|}
\hline \multirow[t]{2}{*}{ Evaluated parameter } & \multicolumn{4}{|c|}{ Rating, $n$ (\%) } \\
\hline & Yes & No & $5-3$ & $2-1$ \\
\hline Satisfaction with overall surgical outcome & $49(89)$ & $6(11)$ & - & - \\
\hline Do you regret your decision about breast reconstruction? & $5(9)$ & $50(91)$ & - & - \\
\hline Would you recommend breast reconstruction to a friend? & $50(91)$ & $5(9)$ & - & - \\
\hline Do you have any limitations in daily activities after reconstruction? & $23(42)$ & $32(58)$ & - & - \\
\hline $\begin{array}{l}\text { Do you have any complaints about discomfort in the reconstructed breast } \\
\text { during intensive exercise? }\end{array}$ & $33(60)$ & $22(40)$ & - & - \\
\hline How much touch sensation in the reconstructed breast is preserved? & - & - & $36(65)$ & $19(35)$ \\
\hline
\end{tabular}


Table 2. Detailed aesthetic satisfaction $(N=55)$

\begin{tabular}{|c|c|c|}
\hline \multirow[t]{2}{*}{ Evaluated parameter } & \multicolumn{2}{|c|}{ Rating, $n(\%)$} \\
\hline & $1-2$ & $3-5$ \\
\hline Symmetry of breasts & $25(45)$ & $30(55)$ \\
\hline Satisfaction with scars & $7(13)$ & $48(87)$ \\
\hline Satisfaction with appearance in clothes & $4(7)$ & $51(93)$ \\
\hline Satisfaction with appearance in close-fitting or revealing clothes (e.g. swimming suit) & $15(27)$ & $40(73)$ \\
\hline Satisfaction with appearance in bra & $10(18)$ & $45(82)$ \\
\hline Satisfaction with nude appearance. & $30(55)$ & $25(45)$ \\
\hline Satisfaction with reconstructed breast size. (inserted implant size) & $9(16)$ & $46(84)$ \\
\hline How natural does your breast look after reconstruction? & $24(44)$ & $31(56)$ \\
\hline How much do you feel the reconstructed breast as a natural part of your body? & $17(31)$ & $38(69)$ \\
\hline
\end{tabular}

average limitations in activities were evaluated as 2.5 in the scale from one to four, which is approximately $62 \%$ [9]. Discomfort could be associated with breast muscle separation from the chest wall creating a pocket for the implant, which can affect the range of motion in the shoulder joint. Involving the pectoral muscle in exercises, the implant beneath it gets squeezed and promotes a feeling of a foreign body and discomfort. It should be admitted that after tissue flap IBR discomfort during intensive exercise is much rarer. For example, after deep inferior epigastric artery perforator flap breast reconstruction only $10 \%$ of patients reported daily life limitations [7].

Half of the patients included in this study were satisfied with breast symmetry, which is comparable to the Beak et al. results - 38\% after direct-to-implant and 65\% after two-stage reconstructions [4]. The results could be improved by using additional correction procedures, such as opposite breast symmetrization, which was not performed in any of our patients with unilateral IBR.

Patients were very satisfied with their appearance in various clothes, and almost all were satisfied with their appearance in daily clothes. Satisfaction of appearance in revealing clothes and in a bra was slightly lower but also remained

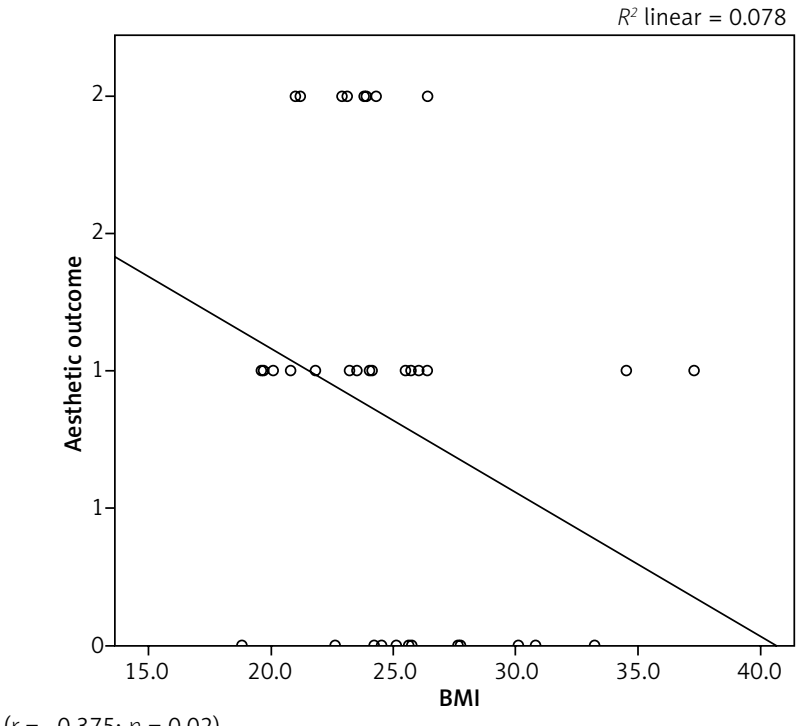

$p=0.02$

Fig. 1. Correlation between body mass index and aesthetic outcome high. Those results were similar to international study results where satisfaction with appearance in clothes was rated between 80 and $92 \%$ and in a bra $74-84 \%$, both after implant-based and tissue flap reconstructions [4, 7-10].

Meanwhile satisfaction with nude appearance in the whole group was significantly lower compared to other studies, whereas satisfaction remained high among women with a spared nipple. Average satisfaction with this parameter in the Dieterich et al. study was 2.44 on a scale from 1 to 4 or $61 \%$ and $74 \%$ in the Beak et al. study $[4,9]$. A potential explanation of the results could be the high rate of skin-sparing mastectomies, 33/55 (\%) in our cohort, and only two of them had some kind of nipple reconstruction in a later stage.

The plastic surgeon rated the overall aesthetic result as positive in more than half of cases, which is comparable with the Vardmanian et al. average result of 2.87 on a scale from 1 to 4, which is approximately 72\%. Symmetry was positively rated in almost the same percentage of cases as in the study conducted by Vardmanian et al. - 74\% [11]. These results show that also in a small breast unit with only implant-based IBR available it is possible to provide IBR with a satisfactory overall and aesthetic outcome. Introduction of nipple sparing mastectomy has contributed considerably

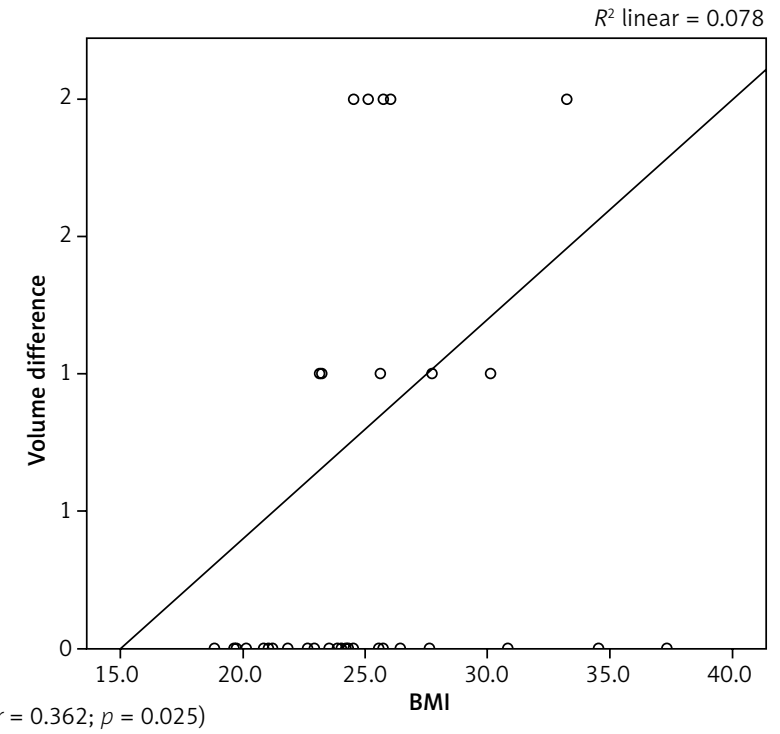

Fig. 2. Correlation between body mass index and volume difference 


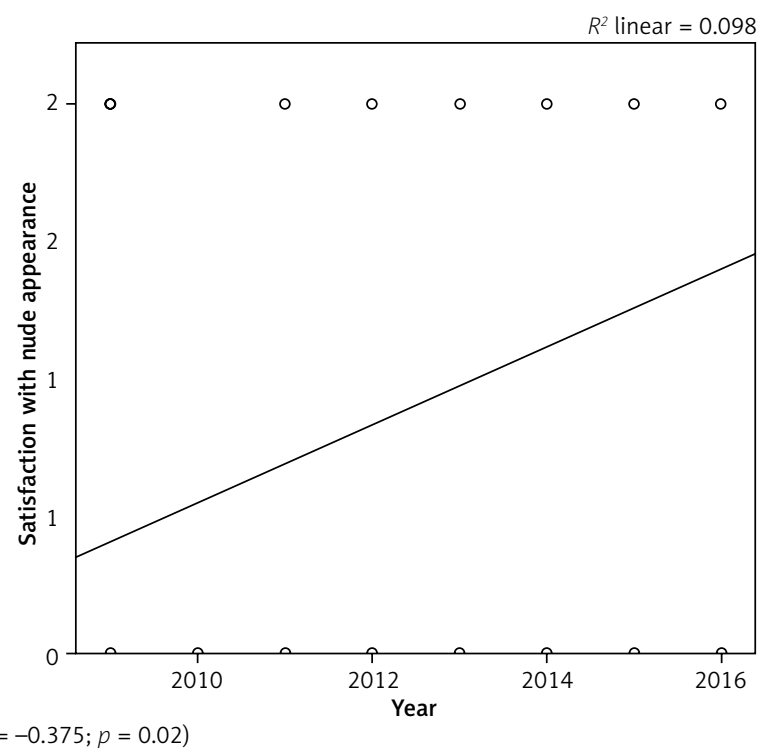

Fig. 3. Level of satisfaction in nude appearance in later cases compared to earlier ones

to patient satisfaction in nude appearance. However, cases with increased $\mathrm{BMI}$ are particularly challenging to achieve good aesthetic satisfaction and volume symmetry with implant-based IBR only. This finding also underlines the need of autologous immediate breast reconstruction availability in the unit for optimal patient satisfaction.

\section{Conclusions}

It is possible to achieve good aesthetic results with implant-based IBR as most of the patients were satisfied with the reconstruction outcome and would recommend it to a friend. However, implant-based IBR revealed suboptimal cosmetic results in the subset of cases with increased $\mathrm{BMI}$ and this finding also underlines the need of contralateral symmetrization procedures and autologous immediate breast reconstruction availability in the unit for optimal patient satisfaction. Nipple preservation considerably contributes to improved aesthetic outcome.

\section{Acknowledgments}

This work was supported by State Research Program "Biomedicine for the public health (BIOMEDICINE)" project 5 "Personalised cancer diagnostics and treatment effectiveness evaluation".

The authors declare no conflict of interest.

\section{References}

1. Filiberti A, Rimoldi A, Callegari M, Nava M, Zanini V, Grisotti A. Immediate versus delayed breast reconstruction. A psychological answer. Eur J Plast Surg 1990; 13: 55.

2. Park SH, Han W, Yoo TK, Lee HB, Jin US, Chang H, Minn KW, Noh DY. Oncologic Safety of Immediate Breast Reconstruction for Invasive Breast Cancer Patients: A Matched Case Control Study. J Breast Cancer 2016; 19: 68-75.

3. Alderman AK, Kuhn LE, Lowery JC, Wilkins EG. Does patient satisfaction with breast reconstruction change over time? Two-year

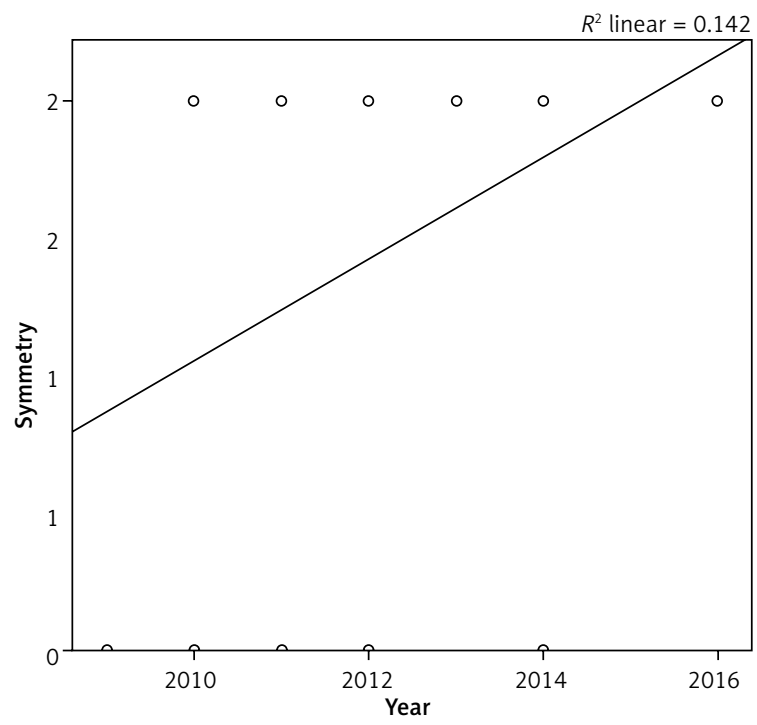

$(r=0.362 ; p=0.025)$

Fig. 4. Symmetry evaluation by plastic surgeon in later cases compared to earlier ones

results of the Michigan Breast Reconstruction Outcomes Study. J Am Coll Surg 2007; 204: 7-12.

4. Baek WY, Byun IH, Kim YS, Lew DH, Jeong J, Roh TS. Patient Satisfaction with Implant Based Breast Reconstruction Associated with Implant Volume and Mastectomy Specimen Weight Ratio. J Breast Cancer 2017; 20: 98-103.

5. Basta MN, Gerety PA, Serletti JM, Kovach SJ, Fischer JP. A Systematic Review and Head-to-Head Meta-Analysis of Outcomes following Direct-to-Implant versus Conventional Two-Stage Implant Reconstruction. Plast Reconstr Surg 2015; 136: 1135-1144.

6. Qureshi AA, Odom EB, Parikh RP, Myckatyn TM, Tenenbaum MM. Patient-Reported Outcomes of Aesthetics and Satisfaction in Immediate Breast Reconstruction After Nipple-Sparing Mastectomy With Implants and Fat Grafting. Aesthet Surg J 2017; 37: 999-1008.

7. Damen TH, Timman R, Kunst EH, Gopie JP, Bresser PJ, Seynaeve C, Menke-Pluijmers MB, Mureau MA, Hofer SO, Tibben A. High satisfaction rates in women after DIEP flap breast reconstruction. J Plast Reconstr Aesthet Surg 2010; 63: 93-100.

8. Struckmann V, Peek A, Wingenbach O, Harhaus L, Kneser U, Holle G. The free fasciocutaneous infragluteal (FCI) flap: Outcome and patient satisfaction after 142 breast reconstructions. J Plast Reconstr Aesthet Surg 2016; 69: 461-469.

9. Dieterich M, Angres J, Stubert J, Stachs A, Reimer T, Gerber B. Patient-Reported Outcomes in Implant-Based Breast Reconstruction Alone or in Combination with a Titanium-Coated Polypropylene Mesh - A Detailed Analysis of the BREAST-Q and Overview of the Literature. Geburtshilfe Frauenheilkd 2015; 75: 692-701.

10. Venus MR, Prinsloo DJ. Immediate breast reconstruction with latissimus dorsi flap and implant: audit of outcomes and patient satisfaction survey. J Plast Reconstr Aesthet Surg 2010; 63: 101-105.

11. Vardanian AJ, Clayton JL, Roostaeian J, Shirvanian V, Da Lio A Lipa JE, Crisera C, Festekjian JH. Comparison of implant-based immediate breast reconstruction with and without acellular dermal matrix. Plast Reconstr Surg 2011; 128: 403e-410e.

\section{Address for correspondence}

\section{Stanislavs Ukleikins}

Department of Surgery

Rīga Stradinšs University

13 Pilsonu iela

Rīga LV-1002, Latvia

e-mail: uk.stanislavs@gmail.com

Submitted: 8.03.2019

Accepted: 8.05.2019 